\title{
Mitochondrial Metabolism - Neglected Link of Cancer Transformation and Treatment
}

\author{
Pokorný J. ${ }^{1}$, Jandová A. ${ }^{1}$, Nedbalová M. ${ }^{2}$, Jelínek F. ${ }^{1}$, Cifra M. ${ }^{1}$, \\ Kučera O. ${ }^{1}$, Havelka D. ${ }^{1,3}$, Vrba J. ${ }^{3}$, Vrba J. Jr. ${ }^{3}$, Čoček A. ${ }^{4}$, Kobilková J. ${ }^{5}$ \\ ${ }^{1}$ Institute of Photonics and Electronics, Academy of Sciences of the Czech Republic, \\ Prague, Czech Republic; \\ ${ }^{2}$ Institute of Physiology, First Faculty of Medicine, Charles University in Prague, \\ Prague, Czech Republic; \\ ${ }^{3}$ Department of Electromagnetic Field, Faculty of Electrical Engineering, Czech \\ Technical University, Prague, Czech Republic; \\ ${ }^{4}$ Department of Otorhinolaryngology, Third Faculty of Medicine, Charles University \\ in Prague, Prague, Czech Republic; \\ ${ }^{5}$ Department of Obstetrics and Gynaecology, First Faculty of Medicine, Charles \\ University in Prague and General University Hospital in Prague, Prague, Czech \\ Republic
}

Received December 23, 2011; Accepted April 11, 2012.

Key words: Physical activity of cells - Cancer - Glycolytic phenotype Mitochondria - Microtubules

\begin{abstract}
Physical processes in living cells were not taken into consideration among the essentials of biological activity, regardless of the fact that they establish a state far from thermodynamic equilibrium. In biological system chemical energy is transformed into the work of physical forces for various biological functions. The energy transformation pathway is very likely connected with generation of the endogenous electrodynamic field as suggested by experimentally proved electrodynamic activity of biological systems connected with mitochondrial and microtubule functions. Besides production of ATP and GTP (adenosine and guanosine triphosphate) mitochondria form a proton space charge layer,
\end{abstract}

This study was supported by grant No. P102/11/0649 of the Czech Science Foundation GA CR.

Mailing Address: Ing. Jiř́ Pokorný, DSc., Institute of Photonics and Electronics, Academy of Sciences of the Czech Republic, Chaberská 57, 18251 Prague 8, Czech Republic; Phone: +420 266773 432; Fax: +420 284680 222;

e-mail: pokorny@ufe.cz 
strong static electric field, and water ordering around them in cytosol - that are necessary conditions for generation of coherent electrodynamic field by microtubules. Electrodynamic forces are of a long-range nature in comparison with bond and cohesive forces. Mitochondrial dysfunction leads to disturbances of the electromagnetic field; its power and coherence may be diminished, and frequency spectrum altered. Consequently, defective electrodynamic interaction forces between cancer and healthy cells may result in local invasion of cancer cells. Further deformation of interaction forces connected with experimentally disclosed spatial disarrangement of the cytoskeleton and disordered electrodynamic field condition metastatic process. Cancer therapeutic strategy targeting mitochondria may restore normal physiological functions of mitochondria and open the apoptotic pathway. Apoptosis of too much damaged cancer cells was observed. Considerable experience with DCA (dichloroacetate) cancer treatment in humans was accumulated. Clinical trials should assess DCA therapeutic potential and collect data for development of novel more effective drugs for mitochondrial restoration of various cancers.

\section{Introduction}

Transformation of chemical energy into useful work in biological systems is not yet fully explained. Due to physical processes and energy supply biological systems are far from thermodynamic equilibrium. The heart, the brain, and the liver are the biggest energy consumers, however, all cells need energy for organization, transport, interactions, and information transfer. Assumption that such an activity is a result of thermal randomness is untenable. In the macroscopic world heat (e.g. based on the expansion of steam or combustion gases) and electric and electromagnetic fields are used for the transformation. The main transformation pathway in biology seems to be based on electrodynamic processes. Any deformation of the transformation pathway causes pathological state that may extend from mild to fatal. Biological activity depends on various physical mechanisms, for instance on polarization of dielectric particles, attraction and repulsion electrodynamic interaction forces, photon information transfer etc.

Physical properties of cells and tissues have been examined in diagnostics and employed for therapy for almost whole timeline of modern medicine; however, physical processes in living cells have been oftentimes simultaneously disregarded as a part of biological activity. As a result of the effort to understand mechanisms underlying dynamic functionality of living matter, attention is now being turned to physics that underpin biological functions. This is especially true for the case of the cancer problem. We have learned from the pathophysiology of malignant cells that they exhibit several properties which contra-distinguish them from healthy cells. Using historical perspective, they exhibit disordered morphology, alteration of stiffness, mitochondrial dysfunction, etc. Many of these properties were described 
in terms of biochemistry and cytology; however, the malignant process hasn't been pictured in its complexity.

For a long time suppression of oxidative metabolism in cancer cells (Warburg et al., 1924; Warburg, 1956) was considered to be an accompanying unimportant effect and not a substantial link along the cancer transformation pathway. Fröhlich predicted coherent electrical polar oscillations and generation of electromagnetic fields in living cells (Fröhlich, 1968a, b, 1969, 1973, 1980) and their disturbances in cancer cells (Fröhlich, 1978). Excitation of the electromagnetic field in living cells was claimed to be impossible due to water viscosity damping (Foster and Baish, 2000) or insufficient energy sources in the cell for excitation (McKemmish et al., 2009; Reimers et al., 2009). The former authors neglected water ordering, the latter authors non-linear properties of the system. Layers of water without solutes observed around microtubules were called clear zones (Amos, 1979). Formation of clear zones was assumed to depend on negative charge at the microtubule surface (Stebbings and Hunt, 1982). Ling (2006) formulated theory of ordering of water molecules in the electrostatic field of the surface charges at the interface. The clear (exclusion) zones were proved to be layers of ordered water (Zheng and Pollack, 2003; Pollack et al., 2006; Zheng et al., 2006). Interfacial water ordering may be formed up to a distance of about $0.1 \mathrm{~mm}$ from the charged surface. lons are excluded from the ordered layer due to strong electric field, thermal fluctuations are diminished as follows from measurement in the wavelength region 3.8-4.6 $\mu \mathrm{m}$, and UV absorbance at $270 \mathrm{~nm}$ increased. The ordered water resembles a gel. Ordered water layers are formed around mitochondria (Pokorný, 2012).

Measurements performed on living cells disclosed electric and electromagnetic oscillations in living cells. Dielectrophoretic forces of the cellular oscillating electric field cause attraction or repulsion of dielectric particles in dependence on their permittivity (Pohl, 1980; Hölzel and Lamprecht, 1994; Hölzel, 2001). Elastic oscillations of the yeast cell membrane in the acoustic range below $2 \mathrm{kHz}$ were measured (Pelling et al., 2004, 2005) and elastic and electric oscillations were compared (Pokorný et al., 2008; Jelínek et al., 2009). Microtubule polymerization in cells may be disrupted by external electromagnetic field at the frequency $0.1-0.3 \mathrm{MHz}$ (Kirson et al., 2004, 2007). Electric oscillations at cellular membrane of yeast and alga cells in the frequency range 1.5-52 MHz were measured (Hölzel and Lamprecht, 1994; Hölzel, 2001). The high values of the electrodynamic activity of synchronized yeast cells in the $M$ phase coincide with the rearrangement of the microtubules into a mitotic spindle, metaphase, and anaphase A and B (Pokorný et al., 2001). Damping of external electromagnetic field caused by cancer tissue at the frequency $465 \mathrm{MHz}$ and its second harmonic was disclosed (Vedruccio and Meessen, 2004). Oscillations in microtubules may be damped in cancer cells by water with decreased level of ordering (Pokorný et al., 2011). Cancer cells display less ordered system (Damadian, 1971). Interactions between cells mediated by 
cellular electromagnetic fields in the red and near-infrared range were observed (Albrecht-Buehler, 1991, 1992, 2005).

Fast polymerization of microtubules in the oscillating electric field was performed (Sahu et al., 2012). Microtubules display resonant oscillations at a frequency about $10 \mathrm{kHz}$ and at several frequencies around $10 \mathrm{MHz}$. The generated electromagnetic signals are frequency and phase locked. Microtubule oscillations have a high quality factor.

In this paper the physical background of the biological activity and malignant transformation pathways will be briefly summarized (Pokorný, 2009a, b; Pokorný et al., 2011) and implications for diagnostics and therapy pointed out. The electromagnetic field measured at living cells is assumed to be generated by essentially non-linear elasto-electrical oscillations in microtubules and that the main conditions for generation of the electrodynamic field are formed by mitochondria. All cancers disturb mitochondrial function and consequently generation of the electrodynamic field too. The cancer treatment modalities should target the disturbances in mitochondria-microtubule cooperating system. Nanotechnological measurements at physiological temperature would disclose basic spectral and power characteristics of electrodynamic oscillations.

\section{Cooperation of mitochondria and microtubules}

Mitochondrial function is not limited to production of ATP and GTP (adenosine and guanosine triphosphate) and to trigger apoptosis. Energy of pyruvate and fatty acids is used for pumping protons into intermembrane space and in this way transformed into electrochemical proton gradient energy. From the intermembrane space protons diffuse into cytosol through the outer membrane that is freely permeable to molecules whose relative molecular mass is 5000 or less. A layer of ordered water and strong static electric field is formed around each functional mitochondrion. Intensity of the static electric field was measured by special fluorescent particles of $30 \mathrm{~nm}$ in diameter (Tyner et al., 2007). The greatest intensity of the electric field (about $3.5 \mathrm{MV} / \mathrm{m}$ ) was measured at the outer mitochondrial membrane. Intensity of the electric field around a single mitochondrion decreases linearly as a function of distance. Significant values were measured even at a distance of $2 \mu \mathrm{m}$ from a mitochondrion. This dependence corresponds to an ordered layer of water around a mitochondrion (Pokorný, 2012). More than $20 \%$ of the cellular volume may be occupied by mitochondria and the ordered water fills up the rest. Cytosol, cytoskeleton, and biological molecules are exposed to a strong electric field.

Production of ATP utilizes electrochemical proton gradient across the inner membrane. ATP is produced with efficiency higher than $40 \%$. The remaining non-utilized energy (nearly 60\%) is liberated from mitochondria as heat, photons (emission of UV photons was detected too), and chemical energy not exploited for ATP and GTP production. 
In the interphase mitochondria are aligned along microtubules, the main organizing structure of the cytoskeleton. Microtubules are composed of tubulin heterodimers that are electric dipoles. Their oscillations generate electrodynamic field (the field in the neighbourhood of the oscillating source whose energy has characteristic features of the electric field; the field may be also called the virtual photon field). The strong electric field around mitochondria can shift microtubule oscillations into highly non-linear region. The electrodynamic field generated by microtubules in the cellular cytoskeleton has to be excited from the cellular energy sources.

Energy supply is an essential condition for oscillations and generation of the electrodynamic field. Energy supply to microtubules is provided by hydrolysis of GTP to GDP (guanosine diphosphate) in the $\beta$ tubulin of the heterodimer after polymerization, by motor proteins moving along microtubules, and by the nonutilized energy liberated from mitochondria. A dominant contribution is very likely formed by the liberated energy.

Biological cellular activity depends on the generated electrodynamic field. Its role in directional transport of mass particles and electrons (Pokorný, 2001; Pokorný et al., 2005a), organization of living matter (Pokorný et al., 2005b), interaction between systems (Pokorný, 2006), and information transfer (Pokorný et al., 2012) was analyzed and published. These works represent a novel contribution to understanding of the biological activity.

Mitochondria and microtubules form a unique cooperating system in the cell (Pokorný, 2009a, b; Pokorný et al., 2011). Figure 1 shows a schematic picture of the mitochondrion and microtubule activity and their cooperation. Mitochondria form conditions for coherent excitation of microtubules by energy supply, low damping, and shift of oscillations into a highly non-linear region.

\section{Cancer disturbances of physical processes}

Suppression of the oxidative production of ATP and GTP and its replacement by fermentative one (Warburg et al., 1924) disturbs all consequent physical processes and biological activity dependent on mitochondrial function. Only about one half of the ATP cell production is covered by mitochondrial supply (Warburg, 1956). One type of mitochondrial dysfunction (called glycolytic phenotype) is caused by inhibition of the pyruvate pathway by PDK - pyruvate dehydrogenase kinase (Bonnet et al., 2007). Mitochondrial dysfunction was found in many types of cancer (Carew and Huang, 2002; Cuezva et al., 2002). Realization of the facts a) that a diverse group of information channels and oncogenes results in mitochondrial dysfunction with increased glycolysis and resistance to apoptosis, b) that the majority of carcinomas have hyperpolarized mitochondria, and c) that most solid tumors have increased glucose uptake is a merit of Michelakis et al. (2008). Consequently, mitochondria targeting treatment may be effective in a large number of diverse malignant tumors, in particular using DCA - dichloroacetate. 


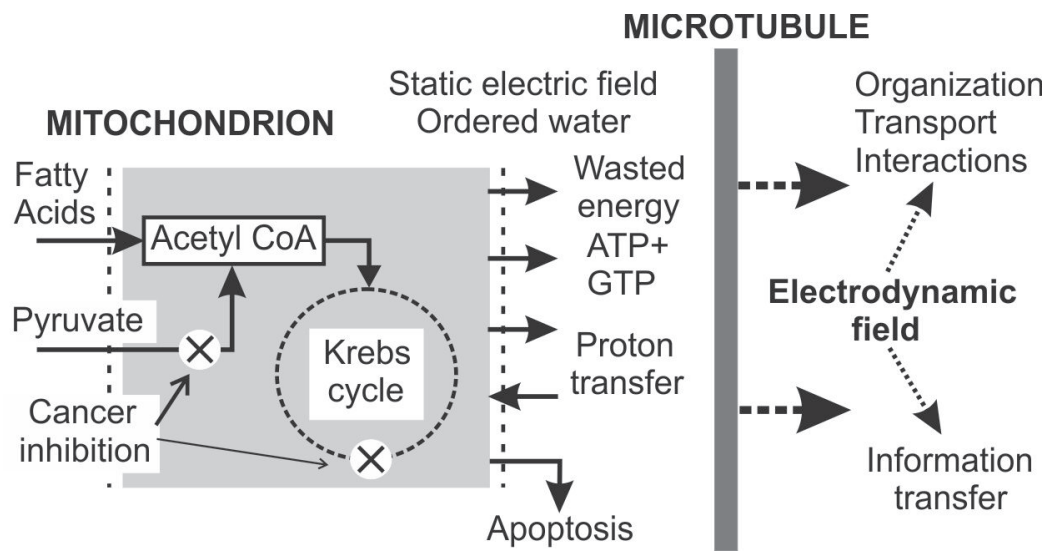

Figure 1 - Cooperation of mitochondria and microtubules in healthy and cancer cells. Transfer of protons from the mitochondrial matrix space into cytosol leads to formation of a strong static electric field and high level of water ordering. Consequently, damping of microtubule oscillations is low. Microtubule oscillations may be excited by energy supply in particular by wasted (non-utilized) energy liberated from mitochondria. Microtubules are electrically polar structures whose oscillations generate electrodynamic field which may participate in organization, transport of molecules and particles, interactions, and information transfer. In cancer cells the level of water ordering is lowered which is caused by mitochondrial dysfunction. Inhibition of the pyruvate pathway in mitochondrion (Bonnet et al., 2007) results in partial suppression of proton transfer from the matrix space (nevertheless, inhibition may be caused also by disturbances in the citric acid cycle - which is pointed at by a thin line with arrow). Cancer cells with blocked pyruvate pathway (i.e. glycolytic phenotype cells) form a large group of cancers.

The lack of mitochondrial hyperpolarization in certain types of cancer, including oat cells lung cancer, lymphomas, neuroblastomas, sarcomas, and some other cancers (Chen, 1988; Michelakis et al., 2008) suggests either a modified glycolytic phenotype or existence of another type(s) of mitochondrial defects and apoptosis blocking. The mitochondrial membrane potential depends also on distribution of ions, for instance $\mathrm{K}^{+}$. By an electrically neutral exchange of protons and potassium ions the $\mathrm{pH}$ gradient decreases and the membrane potential increases (Chen, 1988).

Diversity of cancer origin agents led to a hypothesis, that mitochondrial dysfunction is a primary cause of cancer and biochemical and genetic deviations develop as consequent events (Seyfried and Shelton, 2010). This hypothesis is not proved and some inconsistency with experimental results may be found. In cervical cancer cells mitochondrial dysfunction is observed in the development period from precancerous to cancer cells (measured by immunity response to LDH virus antigen and specific tumor antigen - Jandová et al., 2009), i.e. after biochemical and genetic changes. Mitochondria are the boundary entities between chemical-genetic and physical processes. Mitochondrial dysfunction disturbs essential physical processes in living cells (Pokorný, 2009a, b; Pokorný et al., 2011). 


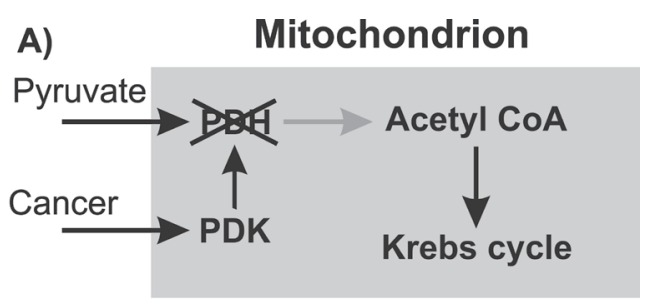

B)

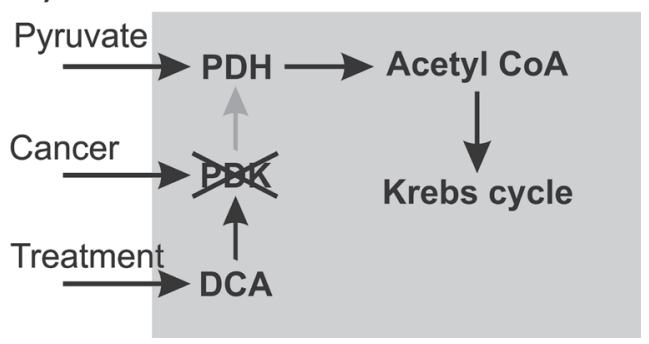

Figure 2 - Effect of DCA on glycolytic phenotype cancer cell. A) The pyruvate pathway is blocked by cancer PDK. PDH (pyruvate dehydrogenase) enzymes in the mitochondrial matrix (the grey area) phosphorylated by PDK are dysfunctional and pyruvate is not broken down into the twocarbon acetyl group on acetyl CoA (Coenzyme A). B) DCA inhibits activity of PDK. Function of PDH enzymes is restored and the pyruvate pathway in mitochondria is open. After Bonnet et al., 2007.

The mitochondrial membrane potential may be assumed to promote both life and death (Michelakis, 2008). Basic processes of life are affected by potential disturbances caused by insufficient energy supply from pyruvate or fatty acids. Activity of pyruvate dehydrogenase (PDH) enzymes is regulated by PDH kinases (PDK-1-PDK-4). Mitochondrial dysfunction in the glycolytic phenotype cancer cell is caused by blocking the pyruvate pathway by the PDH kinases (Figure 2). Hyperpolarization of the mitochondrial inner membrane may be accompanied by low level of water ordering, diminution of the intensity of the static electric field around mitochondrion, decrease of the non-utilized energy efflux, and low expression of the $\mathrm{K}^{+}$channels. DCA disturbs PDK-1, -2, and -4 (Sun et al., 2010) and may restore a normal mitochondrial activity resulting in normal cell function or switching on apoptosis of too aberrant cells. It has to be mentioned that apoptosis is not always associated with mitochondrial depolarization (Bonnet et al., 2007). A need for developing better PDK inhibitors was suggested (McFat et al., 2008). It should also be mentioned that DCA action is based on attacking PDK and not the mechanism of its production.

\section{Disturbances of cytoskeleton}

Microtubules form a structure generating electrodynamic field in the cell. Biological activity may depend on power, frequency spectrum, coherence, and space pattern of the generated electrodynamic field. Geometrical arrangement of microtubules and other cytoskeleton structures determines space pattern of the generated field. Cytoskeleton disturbances are induced along the pathway of cancer cell development before malignant properties are formed. Mechanical properties of healthy and cancer cells of the same tissue (investigated under action of external forces) are significantly different (Cross et al., 2007; Lee and Lim, 2007). 
Deformability of human non tumorigenic epithelial breast cells (MCF-10), of non-metastatic cancer (adenocarcinoma) cells (MCF-7), and increased metastatic potential cells (modMCF-7) is 10, 20, and 30\%, respectively (Guck et al., 2005). Mechanical properties of human pancreatic cells (Pac-1) are altered after application of SPC (sphingosylphosphorylcholine) that plays a critical role in the metastatic invasion of gastrointestinal cancers. The keratin network shrinks around the nucleus, elasticity of the cell is reduced, and energy dissipated by mechanical deformation increased (Beil et al., 2003; Suresh et al., 2005; Suresh, 2007). These effects might be caused by diminished electrodynamic interactions that are longrange in comparison with bond and cohesive ones. Some morphological changes used for cytological and histological evaluation of cancer development may result from cytoskeleton defects. For instance, in the cytological pictures the keratin network shrinkage may be characterized by wrinkling of the nucleus membrane.

Mitochondrial dysfunction may result in lower power and coherence of the generated electrodynamic field in cancer cells in comparison with healthy cells. Attraction forces depend on the power and coherence of the generated electrodynamic field. Attraction forces between cancer cells may be smaller than those between healthy cells or a healthy and a cancer cell. Cancer cells are attracted by the healthy cells around tumor and pulled into healthy tissue. This force effect may constitute essential part of the local invasion (Pokorný, 2006). Perinuclear reorganization of phosphorylated keratin filaments in response to SPC treatment precedes metastatic process (Beil et al., 2003; Suresh et al., 2005; Suresh, 2007). Due to the cytoskeleton reorganization the space pattern of the generated electrodynamic field may be damaged to such extent that the cancer cell can release itself from interaction with surrounding cells, liberate, and make metastases in distant organs. It might be connected with further decrease of the supplied power, coherence, and non-linear properties of microtubules, and disturbance of the frequency spectrum. These mechanisms may be closely conditioned by the extracellular matrix defects.

\section{Treatment of mitochondria in cancer patients}

Considerable clinical experience was accumulated with application of DCA for treatment of cancer patients. Casual reports are available (Khan, 2011; Medicor Cancer Centres in Canada - http://www.medicorcancer.com/). Patients with various tumors (for instance metastatic renal, lung, and ovarian carcinoma) were treated. The doses were from 10 to $25-50 \mathrm{mg} / \mathrm{kg} /$ day in continual and cyclic (1-3 weeks on followed by 1 week off) courses (doses are limited by severity of the side effects). Duration of treatment was at least one month.

Side effects are dose and age dependent. The side effects are mild, not serious, and do not endangering life. The majority of the side effects are of neurological and gastrointestinal origin. Neurological side effect concerns peripheral neuropathy, sedation, fatigue, confusion, hallucination, memory problems, hand 
tremor, and gait disturbances. Gastrointestinal side effects include heartburn, nausea, vomiting, and indigestion. The most dangerous process is the tumor lysogenic syndrome. Large number of cells is rapidly killed and a sudden release of the dead cell material into the blood stream may cause abnormal heart rhythms and kidney failure.

The response to DCA treatment was developed within 2 week if the dose was about $20 \mathrm{mg} / \mathrm{kg} /$ day or higher. For lower doses the response was weaker or delayed. Positive response was experienced by $60-70 \%$ of treated patients. In the positively responded patients DCA may have a palliative effect. However, DCA may have much better responds too. It is important to emphasize, that the aggressive cancer forms were also transformed from a fatal disease to a chronic disease with simple medications. Medicor Cancer Centres announced more than 800 treated patients before October 2011. All the patients were previously treated by standard methods, however, the treatment was either ineffective or could not continue.

\section{Discussion}

Physical processes in living cells depend on mitochondrial function that plays a special role in the cell. Mitochondria are regulated by chemical-genetic messengers but besides triggering apoptosis their activity is mainly connected with physical mechanisms. Mitochondrial function cannot be reduced to energy conversion into ATP and GTP. Transfer of protons from the matrix space into cytosol creates strong static electric field around mitochondria with consequences in non-linear effects in microtubules and water ordering in cytosol. Mitochondria perform an essential role in cell organization and activity. Their dysfunction degrades physical processes. This is the case of cancer transformation. At certain stage of cancer development mitochondrial dysfunction is formed. Chemical, genetic, and physical mechanisms are mutually coupled.

Discovery of microtubule oscillation frequencies may open a way for further understanding of biological activity and its alterations by cancer. Nanotechnological sensors and amplifiers may be used for measurement of electrodynamic activity of healthy and cancer cells in the frequency range around $10 \mathrm{MHz}$ to determine physical differences. It is claimed that the resonant frequencies depend on the secondary protein structure $-\alpha$ helixes, amino-acid groups, and $\beta$ sheets (Sahu et al., 2012). Consequently, all proteins should be able to oscillate at resonant frequencies and electrically polar protein molecules generate electrodynamic field. Based on these considerations long-range interaction between individual proteins may exist. Therefore, drugs interacting attractively with a convenient target may be synthesized. This should hold for cancer treatment by targeting mitochondria too.

Cancer is a complex microevolution, multistep, and multi branched process. Essential life mechanisms are misused and gradually altered by cancer. Adaptability of cancer cells is large. Any "one-point" treatment may be overcome by altered 
cancer mechanism. Therefore, the treatment should be complex, target essential links in different branches along the cancer transformation pathway, based on combination of various chemical compounds, and reproducible in recurrent cases.

Adaptability and heterogeneity of cancer process is an obstacle in its effective treatment. Above it, differences between healthy and cancer cells may be of quantitative type. Cancer treatment based on the therapeutic strategy of killing the cancer cells can destroy the healthy cells too. The negative side effects may be individual and vary from negligible to serious. For instance, the differences in increased fermentative ATP production level may belong to a quantitative type. Cancer therapy based on breaking fermentative production may considerably damage healthy cells too.

Dysfunction of mitochondria is a very remarkable difference between a healthy and a cancer cell. As a result of mitochondrial dysfunction the endogenous electrodynamic field generated in cancer cells has decreased power, coherence, disturbed frequency spectrum and spatial pattern that may be the greatest functional differences in comparison with healthy cells (Pokorný, 2009a, b; Pokorný et al., 2011). Restoration of normal mitochondrial function re-establishes conditions for normal physical processes and unlocks the apoptotic pathway. If the cell is too much damaged, for instance by disorganization of the cytoskeleton or the DNA structure, mitochondria can send a signal to start cell pre-programmed death. Targeting mitochondria very likely acts on the region of fundamental differences between healthy and cancer cells. It may be assumed that therapeutic strategy of cancer treatment should be pointed to restoration of normal mitochondrial function.

The only known effective drug for restoration of normal mitochondrial function in a considerable group of cancers is DCA. DCA inhibits some PDK $(1,2,4)$ blocking pyruvate transfer and its utilization in mitochondrial matrix. A considerable preclinical evidence of DCA effects in vitro and in vivo was accumulated. Experience in human cancer treatment is substantial too. Early clinical trials should be started to determine a possible role of DCA in cancer treatment. First of all tumor reaction and development should be observed and then patient states determined from laboratory tests and measurements. Specific symptoms after DCA application need to be disclosed too. Such clinical trials with DCA may determine specific requirements for future drug development and open the way for a novel strategy in cancer treatment - restoring the normal function of mitochondria and the cell, and unlocking apoptosis.

\section{Conclusion}

Mitochondria are boundary entities between chemical-genetic and physical processes in living cells. They set up conditions for physical mechanisms in living cells. Production of ATP and GTP, liberation of non-utilized energy, establishment of a strong static electric field and formation of layer of ordered water around 
mitochondria are essential conditions for generation of coherent electrodynamic field by microtubules. This electrodynamic field may provide directional transport in the cell, organization of structures and organelles, interactions in the cell and between cells, and information transfer.

Mitochondrial dysfunction in cancer cells may be caused by interruption of pyruvate pathway in the matrix or another defect (for instance in the citric acid cycle) causing diminished proton transfer from the matrix space. Consequently, electrodynamic field generated by microtubules may be characterized by a low power, diminished coherence, and altered frequency spectrum; the space pattern of the field may be disordered too. Biological functions dependent on generated electrodynamic field are disturbed.

Treatment of cancer should be based on essential and specific differences between healthy and cancer cells. The customary cancer therapeutic strategy is based on cancer cell killing. The main issue is aimed at finding sufficiently specific property of cancer cells to limit damage to healthy cells. However, the healthy cells may be damaged too and treatment of recurrent cancers remains unsolved. Mitochondrial dysfunction may be a specific and strong difference between healthy and cancer cells. Treatment by DCA may stimulate synthesis of novel drugs restoring mitochondrial physiological role and normal cell function in various cancers.

\section{References}

Albrecht-Buehler, G. (1991) Surface extensions of 3 T3 cells towards distant infrared light sources. J. Cell Biol. 114, 493-502.

Albrecht-Buehler, G. (1992) Rudimentary form of cellular “vision”. Proc. Natl. Acad. Sci. USA 89, 8288-8293.

Albrecht-Buehler, G. (2005) A long-range attraction between aggregating 3 T3 cells mediated by near-infrared light scattering. Proc. Natl. Acad. Sci. USA 102, 5050-5055.

Amos, L. A. (1979) Structure of microtubules. In: Microtubules, eds. Roberts, K., Hyam, J. S., Pp. 1-64, Academic Press, London, New York.

Beil, M., Micoulet, A., von Wichert, G., Paschke, S., Walther, P., Omary, M. B., Van Veldhoven, P. P., Gern, U., Wolff-Hieber, E., Eggermann, J., Waltenberger, J., Adler, G., Spatz, J., Seufferlein, T. (2003) Sphingosylphosphorylcholine regulates keratin network architecture and visco-elastic properties of human cancer cells. Nat. Cell Biol. 5, 803-811.

Bonnet, S., Archer, S. L., Allalunis-Turner, J., Haromy, A., Beaulieu, Ch., Thompson, R., Lee, Ch. T., Lopaschuk, G. D., Puttagunta, L., Bonnet, S., Harry, G., Hashimoto, K., Porter, Ch. J., Andrade, M. A., Thebaud, B., Michelakis, E. D. (2007) A mitochondria- $\mathrm{K}^{+}$channel axis is suppressed in cancer and its normalization promotes apoptosis and inhibits cancer growth. Cancer Cell 11, 37-51.

Carew, J. S., Huang, P. (2002) Mitochondrial defects in cancer. Mol. Cancer 1, 9-20.

Chen, L. B. (1988) Mitochondrial membrane potential in living cells. Annu. Rev. Cell Biol. 4, 155-181.

Cross, S. E., Jin, Y.-S., Rao, J., Gimzewski, J. K. (2007) Nanomechanical analysis of cells from cancer patients. Nat. Nanotechnol. 2, 780-783.

Cuezva, J. M., Krajewska, M., López de Heredia, M., Krajewski, S., Santamaria, G., Kim, H., Zapata, J. M., Marusawa, H., Chamorro, M., Reed, J. (2002) The bioenergetic signature of cancer: a marker of tumor progression. Cancer Res. 62, 6674-6681. 
Damadian, R. (1971) Tumor detection by nuclear magnetic resonance. Science 171, 1151-1153.

Foster, K. R., Baisch, J. W. (2000) Viscous damping of vibrations in microtubules. J. Biol. Phys. 26, 255-260.

Fröhlich, H. (1968a) Bose condensation of strongly excited longitudinal electric modes. Phys. Lett. A 26, 402-403.

Fröhlich, H. (1968b) Long-range coherence and energy storage in biological systems. Int. J. Quantum Chem. II, 641-649.

Fröhlich, H. (1969) Quantum mechanical concepts in biology. In: Theoretical Physics and Biology, ed. Marois, M., pp. 13-22, North Holland, Amsterdam (Proc. $1^{\text {st }}$ Int. Conf. Theor. Phys. Biol., Versailles, 1967).

Fröhlich, H. (1973) Collective behaviour of non-linearly coupled oscillating fields (with applications to biological systems). J. Collect. Phenom. 1, 101-109.

Fröhlich, H. (1978) Coherent electric vibrations in biological systems and cancer problem. IEEE Trans. MTT 26, 613-617.

Fröhlich, H. (1980) The biological effects of microwaves and related questions. Adv. Electronics Electron Phys. 53, 85-152.

Guck, J., Schinkinger, S., Lincoln, B., Wottawah, F., Ebert, S., Romeyke, M., Lenz, D., Erickson, H. M., Ananthakrishnan, R., Mitchell, D., Käs, J., Ulvick, S., Bilby, C. (2005) Optical deformability as an inherent cell marker for testing malignant transformation and metastatic competence. Biophys. J. 88, 3689-3698.

Hölzel, R. (2001) Electric activity of non-excitable biological cells at radio frequencies. Electro Magnetobiol. 20 , $1-13$.

Hölzel, R., Lamprecht, I. (1994) Electromagnetic fields around biological cells. Neural Network World 4, 327-337.

Jandová, A., Pokorný, J., Kobilková, J., Janoušek, M., Mašata, J., Trojan, S., Nedbalová, M., Dohnalová, A., Beková, A., Slavík, V., Čoček, A., Sanitrák, J. (2009) Cell-mediated immunity in cervical cancer evolution. Electromagn. Biol. Med. 28, 1-14.

Jelínek, F., Cifra, M., Pokorný, J., Vaniš, J., Šimša, J., Hašek, J., Frýdlová, I. (2009) Measurement of electrical oscillations and mechanical vibrations of yeast cells membrane around $1 \mathrm{kHz}$. Electromagn. Biol. Med. 28, 223-232.

Khan, A. (2011) Use of oral dichloroacetate for palliation of leg pain arising from metastatic poorly differentiated carcinoma: a case report. J. Palliat. Med. 14, 1-6.

Kirson, E. D., Gurvich, Z., Schneiderman, R., Dekel, E., Itzhaki, A., Wasserman, Y., Schatzberger, R., Palti, Y. (2004) Disruption of cancer cell replication by alternating electric fields. Cancer Res. 64, 3288-3295.

Kirson, E. D., Dbalý, V., Tovaryš, F., Vymazal, J., Soustie, R., Wasserman, Y., Salzberg, M., Ryffel, B., Goldsher, D., Dekel, E., Palti, Y. (2007) Alternating electric fields arrest cell proliferation in animal tumor models and human brain tumors. Proc. Natl. Acad. Sci. USA 104, 10152-10157.

Lee, G. Y. H., Lim, Ch. T. (2007) Biomechanics approaches to studying human diseases. Trends Biotechnol. 25, $111-118$.

Ling, G. (2006) A new theoretical foundation for the polarized-oriented multilayer theory of cell water and for inanimate systems demonstrating long-range dynamic structuring of water molecules. Physiol. Chem. Phys. Med. NMR 35, 91-130.

McFate, T., Mohyeldin, A., Lu, H., Thakar, J., Henriques, J., Halim, N. D., Wu, H., Schell, M. J., Tsang, T. M., Teahan, O., Zhou, S., Califano, J. A., Jeoung, N. H., Harris, R. A., Verma, A. (2008) Pyruvate dehydrogenase complex activity controls metabolic and malignant phenotype in cancer cells. J. Biol. Chem. 283, 22700-22708.

McKemmish, L. K., Reimers, J. R., McKenzie, R. H., Mark, A. E., Hush, N. S. (2009) Penrose-Hameroff orchestrated objective-reduction proposal for human consciousness is not biologically feasible. Phys. Rev. $E$ Stat. Nonlin. Soft Matter Phys. 80, 021912-1-021912-6. 
Michelakis, E. D. (2008) Mitochondrial medicine. A new era in medicine opens new windows and brings new challenges. Circulation 117, 2431-2434.

Michelakis, E. D., Webster, L., Mackey, J. R. (2008) Dichloroacetate (DCA) as a potential metabolic-targeting therapy for cancer. Br. J. Cancer 99, 989-994.

Pelling, A. E., Sehati, S., Gralla, E. B., Valentine, J. S., Gimzewski, J. K. (2004) Local nano-mechanical motion of the cell wall of Saccharomyces cerevisiae. Science 305, 1147-1150.

Pelling, A. E., Sehati, S., Gralla, E. B., Gimzewski, J. K. (2005) Time dependence of the frequency and amplitude of the local nanomechanical motion of yeast. Nanomedicine: Nanotechnology, Biology, and Medicine 1, 178-183.

Pohl, H. A. (1980) Oscillating fields about growing cells. Int. J. Quantum Chem. Quantum Biol. Symp. 7, $411-431$.

Pokorný, J. (2001) Endogenous electromagnetic forces in living cells: Implications for transfer of reaction components. Electro Magnetobiol. 20, 59-73.

Pokorný, J. (2006) The role of Fröhlich's coherent excitations in cancer transformation of cells. In: Herbert Fröhlich, FRS: A Physicist ahead of His Time, eds. Hyland, G. J., Rowlands, P., pp. 177-207, The University of Liverpool, Liverpool.

Pokorný, J. (2009a) Biophysical cancer transformation pathway. Electromagn. Biol. Med. 28, 105-123.

Pokorný, J. (2009b) Fröhlich's coherent vibrations in healthy and cancer cells. Neural Network World 19, 369-378.

Pokorný, J. (2012) Physical aspects of biological activity and cancer. AIP Adv. 2.

Pokorný, J., Hašek, J., Jelínek, F., Šaroch, J., Palán, B. (2001) Electromagnetic activity of yeast cells in the M phase. Electro Magnetobiol. 20, 371-396.

Pokorný, J., Hašek, J., Jelínek, F. (2005a) Electromagnetic field of microtubules: Effects on transfer of mass particles and electrons. J. Biol. Phys. 31, 401-514.

Pokorný, J., Hašek, J., Jelínek, F. (2005b) Endogenous electric field and organization of living matter. Electromagn. Biol. Med. 24, 185-197.

Pokorný, J., Hašek, J., Vaniš, J., Jelínek, F. (2008) Biophysical aspects of cancer - Electromagnetic mechanism. Indian J. Exp. Biol. 46, 310-321.

Pokorný, J., Vedruccio, C., Cifra, M., Kučera, O. (2011) Cancer physics: diagnostics based on damped cellular elastoelectrical vibrations in microtubules. Eur. Biophys. J. 40, 747-759.

Pokorný, J., Martan, T., Foletti, A. (2012) High capacity optical channels for bioinformation transfer: acupuncture meridians. J. Acupunct. Meridian Stud. 5, 34-41.

Pollack, G., Cameron, I., Wheatley, D. (2006) Water and the Cell. Springer, Dordrecht.

Reimers, J. R., McKemmish, L. K., McKenzie, R. H., Mark, A. E., Hush, N. S. (2009) Weak, strong, and coherent regimes of Fröhlich condensation and their applications to terahertz medicine and quantum consciousness. Proc. Natl. Acad. Sci. USA 106, 4219-4224.

Sahu, S., Ghosh, S., Hirata, K., Fujita, D., Bandyopadhyay, A. (2012) Ultra-fast condensation of tubulins into microtubule unravels a generic resonance engineering. Nat. Mater. (in press)

Seyfried, T. N., Shelton, L. M. (2010) Cancer as a metabolic disease. Nutr. Metab. (Lond.) 7, 7.

Stebbings, H., Hunt, C. (1982) The nature of the clear zone around microtubules. Cell Tissue Res. 227, 609-617.

Sun, R. C., Fadia, M., Dahlstrom, J. E., Parish, C. R., Board, P. G., Blackburn, A. C. (2010) Reversal of the glycolytic phenotype by dichloroacetate inhibits metastatic breast cancer cell growth in vitro and in vivo. Breast Cancer Res. Treat. 120, 253-260.

Suresh, S. (2007) Biomechanics and biophysics of cancer cells. Acta Mater. 55, 3989-4014.

Suresh, S., Spatz, J., Mills, J. P., Micoulet, A., Dao, M., Lim, C. T., Beil, M., Seufferlein, T. (2005) Connections between single-cell biomechanics and human disease states: gastrointestinal cancer and malaria. Acta 
Biomater. 1, 15-30.

Tyner, K. M., Kopelman, R., Philbert, M. A. (2007) "Nanosized voltmeter" enables cellular-wide electric field mapping. Biophys. J. 93, 1163-1174.

Vedruccio, C., Meessen, A. (2004) EM cancer detection by means of non-linear resonance interaction. In: Proceedings PIERS, Progress in Electromagnetics Research Symposium, Pisa, Italy, March 28-31 2004, PP. 909-912.

Warburg, O. (1956) On the origin of cancer cells. Science 123, 309-314.

Warburg, O., Posener, K., Negelein, E. (1924) Über den Stoffwechsel der Carcinomzelle. Biochem. Z. 152, 309-344.

Zheng, J., Pollack, G. H. (2003) Long-range forces extending from polymer-gel surfaces. Phys. Rev. E Stat. Nonlin. Soft Matter Phys. 68, 031408-1-031408-7.

Zheng, J., Chin, W., Khijniak, E., Khijniak, E. Jr., Pollack, G. H. (2006) Surfaces and interfacial water: evidence that hydrophilic surfaces have long-range impact. Adv. Colloid Interface Sci. 127, 19-27. 